\title{
Orchestrating cells on a chip: Employing surface acoustic waves towards the formation of neural networks
}

\author{
Manuel S. Brugger, ${ }^{1}$ Sarah Grundeen, ${ }^{2,3,4}$ Adele Doyle, ${ }^{3,4,5}$ Luke Theogarajan, ${ }^{2,4,5}$ \\ Achim Wixforth, ${ }^{1,6,7}$ and Christoph Westerhausen ${ }^{1,6,7, *}$ \\ ${ }^{1}$ Chair for Experimental Physics 1, University of Augsburg, 86159 Augsburg, Germany \\ ${ }^{2}$ Department of Electrical and Computer Engineering, University of California, Santa Barbara, Santa Barbara, California, 93106, USA \\ ${ }^{3}$ Neuroscience Research Institute, University of California, Santa Barbara, Santa Barbara, California 93106-5060, USA \\ ${ }^{4}$ Center for Bioengineering, University of California, Santa Barbara, Santa Barbara, California 93106-5170, USA \\ ${ }^{5}$ California NanoSystems Institute, University of California, Santa Barbara, Santa Barbara, California 93106-6105, USA \\ ${ }^{6}$ Nanosystems Initiative Munich (NIM), Schellingstraße 4, 80799 Munich, Germany \\ ${ }^{7}$ Augsburg Center for Innovative Technologies (ACIT), 86159 Augsburg, Germany
}

(Received 4 March 2018; published 18 July 2018)

\begin{abstract}
For the investigation of cell-cell interaction in general and for neural communication and future applications of neural networks, a controllable and well-defined network structure is crucial. We here propose the implementation of an acoustically driven system for tunable and deliberate stimulation and manipulation of cell growth on a chip. This piezoelectric chip allows us to generate a checkerboard-like standing surface acoustic wave pattern coupled to a fluid layer in a microfluidic chamber on top. Such a dynamically induced patterning lattice is shown to allow for the active positioning of the neurons and subsequent guided neurite outgrowth, thus finally overcoming the limitations of static approaches. After thorough characterization of the resulting tunable potential landscape, we successfully demonstrate cell adhesion and even growth of the such positioned cells within the well-defined pressure nodes. We demonstrate neuron growth at predetermined positions and observe a subsequent neurite outgrowth, even being correlated with the artificial potential landscape. For the very delicate and sensitive primary neural cells, this is a change of paradigm! Our experimental findings give us confidence that our hybrid lab-on-a-chip system in the near future will allow researchers to study cell-cell interaction of primary neurons. If scaled to a true network level, it will enable us to control and study how neural networks connect, interact, and communicate.
\end{abstract}

DOI: 10.1103/PhysRevE.98.012411

\section{INTRODUCTION}

Since the creation of the first neuron-silicon interface by Fromherz in 1991 [1], the integration of large-scale neural and electronic circuitry has been a targeted objective worldwide. The neuronal part of hybrid neural networks is boldly envisioned to take over tasks like learning, associative memory, and pattern recognition [2]. However, such complex tasks require, at minimum, a well-defined neural network [3]. In the past, there have been many attempts to improve the complexity of engineered neural networks by, e.g., employing lanes of extracellular matrix proteins (EMPs) or micro- and nanopillars for cell containment [3-5]. So far, however, the more or less random seeding process and very static nature of most of the experimental setups severely impeded the creation of a deliberate and controlled distribution of cells. On patterned EMPs, for example, neuronal extensions after about one day begin to also reach out into noncoated sections [4]. These challenges leave most of the current issues like large-scale patterning of neurons on a silicon chip and the controlled outgrowth of the neuronal extensions unsolved. We thus here propose that by employing acoustic force fields, dynamically

\footnotetext{
*christoph.westerhausen@gmail.com
}

tunable in space and time, in combination with state-of-the-art microfluidics on a chip, a fully controllable cell patterning and cell guidance is now within reach.

Surface acoustic waves (SAWs) are well established and have entered industry in many technological fields. About 15 years ago, SAW-driven microfluidic and biological applications were also developed and taken up by many different research groups worldwide [6-12]. Here we rely on Rayleigh waves, a distinct mode of SAWs, which exhibit a surface elongation of the substrate that is orthogonal to the propagation direction and normal to the surface [13]. By interfering multiple SAWs, we create standing wave patterns (SSAWs) on the substrate and correspondingly within the adjacent fluid layer. This two-dimensional pressure lattice is then employed to align single cells in suspension. By precisely controlling the temperature, $\mathrm{pH}$, and culture media, we ensure proper cell growth conditions. So far, the deliberate and on-demand patterning of only nonliving particles or comparatively simple red blood cells within a fluid have been reported $[10,14,15]$. For example, Guo et al. presented another approach to control cell-cell interactions using SAWs and demonstrated a tunable intercellular distance [11]. Recent reports on patterning of cells in hydrogel environments claim three-dimensional patterning but cannot pattern single cells [16]. However, to our knowledge, there are no reports yet on the deliberate and controlled 
adhesion and growth of cells after patterning or even culturing neurons on a SAW-driven. Here we show surviving primary neurons, patterned by a dynamical SAW-based approach and forming even networks, where the neurite directions correlate with the minima of the applied potential landscape. We thus envision that the technique will prove valuable to create welldefined neural networks in the near future without facing challenges described above.

\section{CHARACTERIZATION OF THE TUNABLE POTENTIAL LANDSCAPE}

Figures 1(a) and 1(b) show the designed experimental setup. For a first check and inspection of the resulting standing wave pattern of the SSAW lattice, we measured the dynamically induced movement of the chip surface by using an atomic force microscope (AFM) in the constant-force-contact mode. Using the naked, dry SAW chip, without the polydimethylsiloxane microchannel the lateral deflection of a cantilever tip provides information about the friction between the surface and the tip. In the displayed $100 \mu \mathrm{m} \times 100 \mu \mathrm{m}$ section the desired standing wave pattern is clearly seen [Fig. 1(c)] for a power level per interdigital transducer (IDT) pair of $P_{\mathrm{IN}}=21 \mathrm{dBm}$. For visualization of the standing acoustic force field and its alignment effect on small objects inside a fluidic microchannel, lateral SAW patterning experiments were performed using polystyrene beads, although it should be noted that they exhibit a more favorable acoustic contrast than the cells (a)

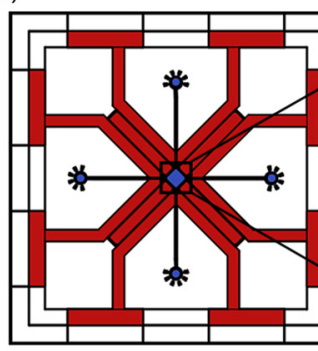

(d)

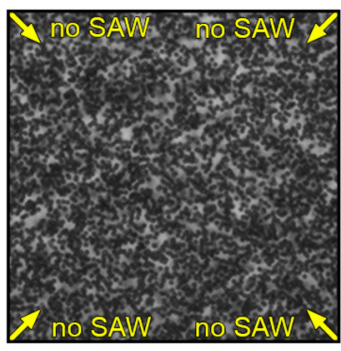

(e)

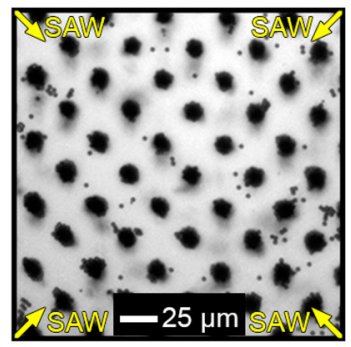

(g)

(h)

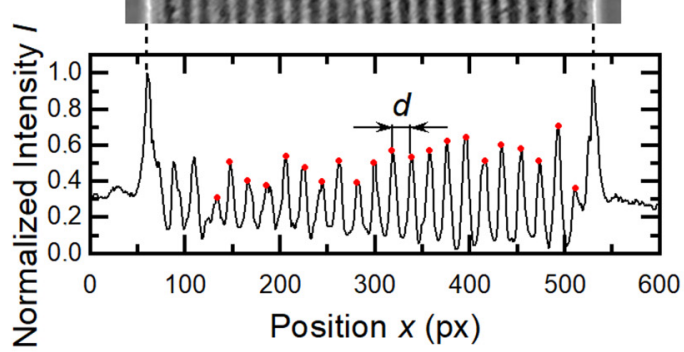

(b)

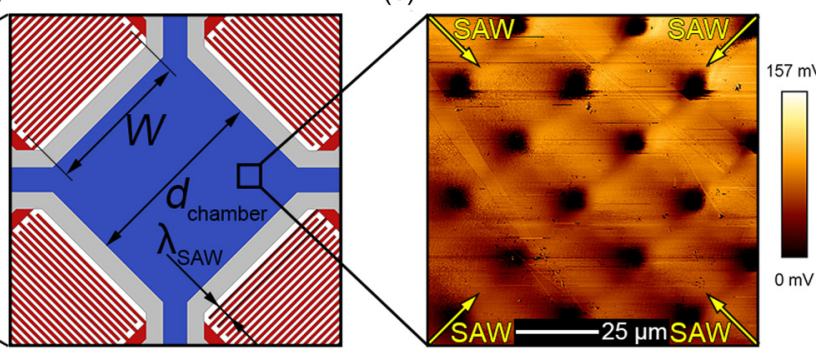

(j)

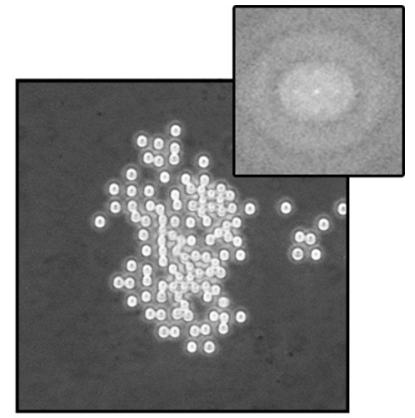

$\mathrm{f}_{\text {SAW }}=239 \mathrm{MHz}$

$\lambda_{\text {SAW }}=15 \mu \mathrm{m}$

(f)

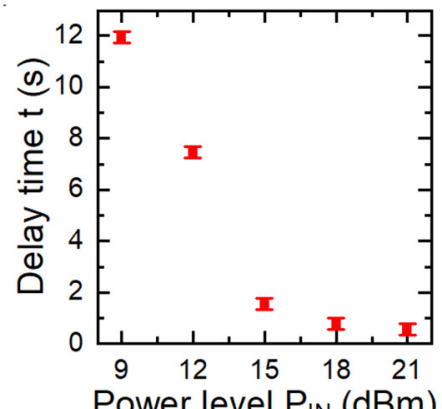

(i)

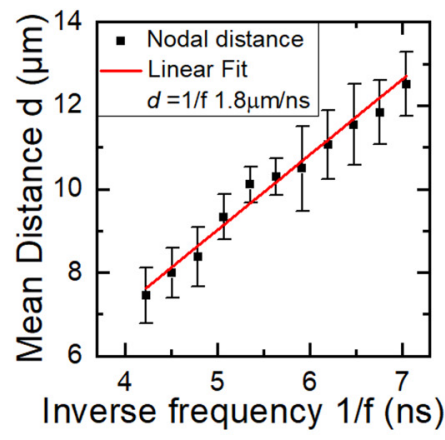

(k)

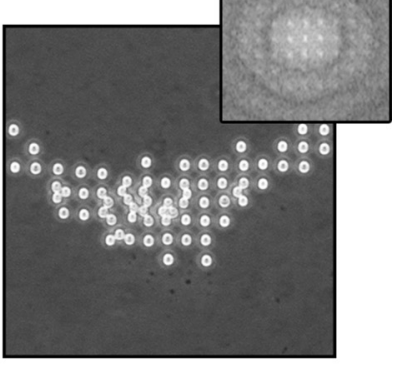

(I)

$$
\begin{aligned}
f_{\text {SAW }} & =197 \mathrm{MHz} \\
\lambda_{\text {SAW }} & =18 \mu \mathrm{m}
\end{aligned}
$$

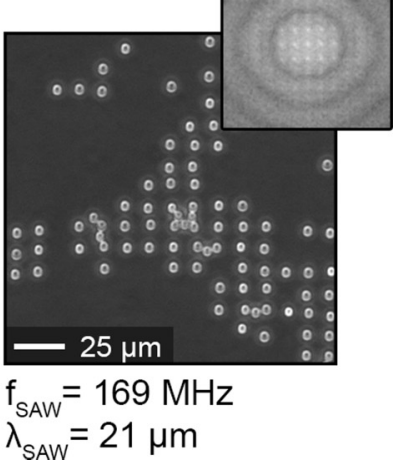

FIG. 1. The experimental setup, dynamically variable in space in time to allow single-particle trapping to align neurons separately. (a), (b) Technical drawing of the SAW chip with an aligned microchannel. (c) AFM micrograph of the SSAW force field. (d-f) SAW-mediated alignment of $d=2 \mu \mathrm{m}$ diameter polystyrene beads $\left(P_{\mathrm{IN}}=12 \mathrm{dBm}\right)$. Starting from a homogenous suspension at $t=0 \mathrm{~s}(\mathrm{~d})$, after $t=21 \mathrm{~s}$, the bead accumulation reached a total maximum patterning efficiency (e). (f) Time to accumulate the beads as a function of the applied SAW power level $P_{\mathrm{IN}}$. (g) Particle accumulation using a "chirped" IDT $\left(d=300 \mathrm{~nm}, f_{\mathrm{SAW}}=147.9 \mathrm{MHz}\right)$. (h) Intensity along the red line in $D$. (i) Nodal distance as a function of the $f_{\mathrm{SAW}}$. (j)-(1) Single-particle trapping of $d=7.38 \mu \mathrm{m} \mathrm{SiO} \mathrm{S}_{2}$ beads with a FFT inset. For $\frac{\lambda_{\mathrm{SAW}}}{D} \geqslant 2.4$ the trapping for single particles is achieved. 
which we consider later. To characterize the power-dependent timescale for patterning, we here used a suspension of $d=$ $2 \mu \mathrm{m}$ diameter beads, suspended in water. We investigate the alignment of the objects as a function of SAW power, $P_{\mathrm{IN}}$, which in turn creates a nearly two-dimensional lateral pressure landscape within the fluid for the given channel height and SAW wavelength. Under the influence of and in interaction with the acoustic body force field, the beads of the initially homogenous suspension [Fig. 1(d)] quickly accumulate in the pressure nodes [Fig. 1(e)]. Increasing the actuating SAW power level, $P_{\mathrm{IN}}$, resulted in an increase of the interaction strength and consequently decreases the time for particles to accumulate at nodal positions [Fig. 1(f)].

In addition to control of the temporal patterning dynamics, the exact lateral position of the SSAW nodes on the chip surface also is tunable by changing the relative phases of the SAW with respect to the fixed coordinate system given by the microchannel geometry. By defining a gradient in the pitch of the IDTs, which determine the wavelength, SAWs over a wide range of frequencies can be excited. Such IDTs are usually referred to as "chirped" transducers [17] in the radio frequency (rf) industry. The nodal positions in the standing wave patterns are then controlled by changing the applied frequency of the rf signal. In our chip, we cover the wavelength range $\lambda_{\min }=15-25 \mu \mathrm{m}$ with a step size of $\Delta \lambda=1 \mu \mathrm{m}$. The chirped IDT aperture was $W=300 \mu \mathrm{m}$, and the height of the PDMS microchannel was $h=25 \mu \mathrm{m}$. For the sake of a better visualization of the nodal positions, in this case only one of the two opposing IDT pairs was activated at a constant power level of $P_{\mathrm{IN}}=18 \mathrm{dBm}$. In this experiment and for demonstration purposes, we are here not generating a two-dimensional dot array but instead a series of parallel one-dimensional lines. We show the SAW-driven accumulation of beads for all excitable frequencies in the range of $f_{\mathrm{SAW}}=141.9-236.5 \mathrm{MHz}$. To illustrate this nice and versatile frequency-adjustable position of acoustic particle accumulation sites, we depict a typical example in Fig. 1(g). In Fig. 1(h) the intensity of the grayscale image in Fig. 1(g) is analyzed along the red line. The average distance $d$ between two adjacent peaks corresponds to the distance between the antinodes. Given the spacing of subsequent lines, an increase of the rf frequency leads to a linear decrease of the nodal distance $d=\frac{c_{\text {Nodal }}}{f}$. As shown in Fig. 1(i), we can confirm this well-known and theoretically predicted linear relation by plotting the experimentally measured distance between two adjacent nodes against the inverse frequency. The slope of the linear fit with $c_{\text {Nodal }}=1803 \frac{\mathrm{m}}{\mathrm{s}}$ is nearly exactly one half of the material's speed of sound for the chosen propagation direction and crystal cut $\left(c_{\mathrm{LiNbO} 3 ;} 128^{\circ}\right.$ rot; $\mathrm{Y}$ cut; $\left.45^{\circ}=3547 \frac{\mathrm{m}}{\mathrm{s}}\right)$. This is consistent with distance $d=\frac{\lambda}{2}$ as the spacing between two adjacent nodes. This elegant and very reliable frequency shift technique now opens up the possibility to create an on-demand and tailored spatiotemporal energy-potential landscape on the chip where the assembly time and the final positions of the particle accumulation spots (or lines) can be adjusted at will.

In effort to approach our main goal of acoustically creating a well-defined neural network on a SAW chip, we focus our work on exploring the possibility of especially single cell manipulation. In a similar approach, Collins et al. recently described one-cell-per-well (OCPW) trapping in an acoustic field [10] if the ratio between acoustic wavelength, $\lambda_{\text {SAW }}$, and the particle's diameter, $D$, lies within the relatively narrow interval $3.2 \leqslant \frac{\lambda \mathrm{saW}}{D} \leqslant 3.6$ for polystyrene beads. Due to the strong correlation between the driving forces, material properties, and acoustic contrast of any SAW-trapped objects, it turns out that soft objects like cells can be OCPW trapped within the range of $2.5 \leqslant \frac{\lambda_{\mathrm{sAW}}}{D} \leqslant 4.0$. To check the applicability of these ranges in the present study, we first investigated the OCPW trapping efficiency of single $\mathrm{SiO}_{2}$ beads $(D=7.38 \mu \mathrm{m})$ using a chirped IDT. By applying different frequencies, we tested the ability of the chip to meet OCPW trapping as shown in Figs. 1(j)-(l) for different SAW frequencies and their associated wavelengths $\left(\lambda_{\mathrm{SAW}}=15-21 \mu \mathrm{m}\right)$. High-frequency stimulation $\left(f_{\mathrm{SAW}}=\right.$ $239 \mathrm{MHz} ; \lambda_{\mathrm{SAW}}=15 \mu \mathrm{m} ; \frac{\lambda_{\text {SAW }}}{D} \approx 2.0$ ), results in accumulation of beads [Fig. 1(j)], whereas single-particle alignment can be observed for lower frequencies starting from the value of $f_{\mathrm{SAW}}=197 \mathrm{MHz}\left(\lambda_{\mathrm{SAW}}=18 \mu \mathrm{m} ; \frac{\lambda_{\mathrm{SAW}}}{D} \approx 2.4\right)$ [Figs. $1(\mathrm{k})$ and 1(1)]. For an analysis of the patterning efficiency, a fast Fourier transformation [see inset images in Figs. 1(j)-1(1)] was performed showing a clear increase in the degree of ordering. Compared to previous reports with polystyrene particles [10], we observed in our experiments with $\mathrm{SiO}_{2}$ an OCPW trapping for even lower ratios $\left(\frac{\lambda_{\mathrm{SAW}}}{D} \approx 2.4\right)$.

Thus, using this single chirped IDT chip, the parameters of the applied rf signal $\left(f_{S A W}\right.$ and $\left.P_{I N}\right)$ can be in fact precisely adjusted to the respective size of the particles to be aligned. This is an important prerequisite to fulfill the conditions for OCPW trapping in contrast to earlier reports [16].

\section{NEURON GROWTH UNDER SAW TREATMENT}

After the characterization of the potential landscape of the on-demand spatiotemporal force fields, we here study adhesion and growth of the very sensitive and delicate neurons. Obviously, all components in the experimental setup have to be carefully chosen to ensure biocompatibility. Moreover, we have to check whether the cells, which are experiencing the SAW-generated body force fields, in fact do survive the treatment. First, we tried to exclude any harmful effects of the ultrasound energy such as cavitation effects or local heating. Thus, and to begin, in very preliminary experiments with living cells under SAW irradiation, we chose not yet to employ classical viability assays, but instead, we checked the cell response directly by observing their adhesion and growth after long-time SAW treatment. For demonstration purposes, we also employed a somewhat simpler SAW chip with only one single IDT $\left(\lambda_{\mathrm{SAW}}=50 \mu \mathrm{m}, W=600 \mu \mathrm{m}\right)$ being oriented normal to the crystal $x$ axis. On top of the SAW chip, a small $(V=1.2 \mathrm{ml})$ PDMS chamber enclosed the cells and the medium. After coating the chip with $V=1 \mathrm{ml}$ polyL-lysine $(1 \mathrm{mg} / 1 \mathrm{ml})$ (PLL), primary neurons were seeded onto the chip at a density of 230 cells $/ \mathrm{mm}^{2}$. Subsequently, the whole experimental setup was incubated $\left(T=37^{\circ} \mathrm{C}, 5 \% \mathrm{CO}_{2}\right)$ for about $6 \mathrm{~h}$ with no SAW being present on the chip. During this time, as expected, primary neurons nicely adhered to the chip and began to grow their neural extensions as being shown in Fig. 2(b). After the successful adhesion, we launched the single, traveling SAW, irradiating the cells with a relatively low SAW power $\left(P_{I N}=6 \mathrm{dBm}\right)$ for $24 \mathrm{~h}$. Cells which did not survive the dissection process, and thus did not adhere, were removed and washed away by the induced acoustic streaming. 


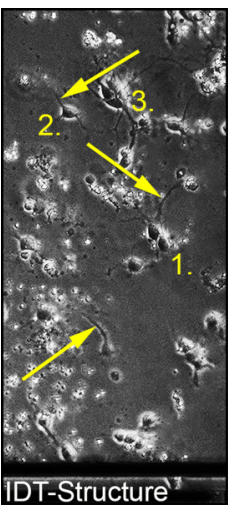

(a) $t=\mathrm{Oh}$

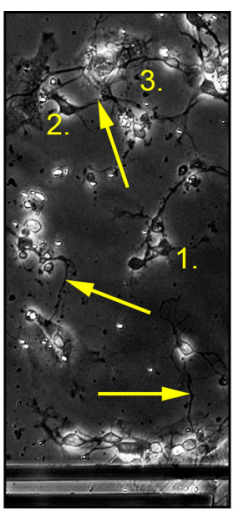

(b) $t=24 h$

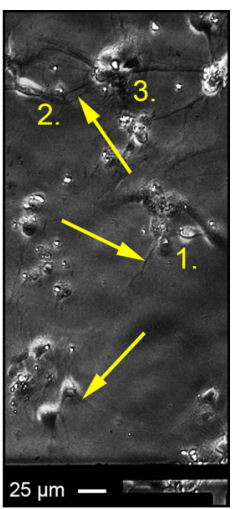

(c) $\mathrm{t}=48 \mathrm{~h}$
FIG. 2. Neuronal cell growth inside the bioreactor to validate that SAW irradiation is not harmful. The numbers denote the same cells at different times. The arrows point at newly grown neurites, indicating healthy cells. (a) No SAW, (b) $P_{\mathrm{IN}}=6 \mathrm{dBm}$, (c), $P_{\mathrm{IN}}=12 \mathrm{dBm}$.

Then we increased the power significantly to $P_{\mathrm{IN}}=12 \mathrm{dBm}$, irradiating the cells under these conditions for another $24 \mathrm{~h}$. The result is shown in Fig. 2(c). Obviously, even after $24 \mathrm{~h}$ of SAW treatment, the cells inside the sound path do not indicate any enhanced apoptosis or induced cell death as compared to the internal reference on the same chip being located well outside the sound path (not shown). During the period of continuous, relatively mild SAW stimulation $\left(P_{\mathrm{IN}}=6 \mathrm{dBm}\right)$, we observed even new neural extensions forming, indicating that the cells continue to grow and interacting with their local environment. Primary neurons thus survive an even quite intense SAW irradiation over a period of $48 \mathrm{~h}$ without any obvious damage. This positive result, obtained for a well-known delicate cell type, is thus consistent with, and even extends beyond, our recent observation for SaOs-2 cells, where SAW treatment of an artificial wound not only proved to be biocompatible, but also resulted in a stimulating effect on cell migration [18].

\section{CONTROLLED CELL ADHESION OF ACOUSTICALLY ALIGNED CELLS}

To verify the actual potential of SSAW to also let cells adhere on a chip in a predetermined pattern, we first employed the immortalized SaOs-2 cell line as a well-studied model representative and then primary neurons. In suspension, $\mathrm{SaOS}$ 2 cells have an average cell diameter of $D=15.2 \pm 1.1 \mu \mathrm{m}$. We thus chose a SSAW-field with $\lambda_{\text {SAW }}=50 \mu \mathrm{m}$. Application of an even larger rf signal, $P_{\mathrm{IN}}=21 \mathrm{dBm}$, ensured initiating cell alignment by creating the desired standing wave potential landscape. Within a second or so, the cells were captured in the resulting body force fields and nicely aligned to the SAW chip nodes. The cells remained trapped in these nodes of the acoustic potential landscape for the whole duration of the SSAW activation. To ensure a sufficient nutrient supply over the course of the experiment, we generated a very small static flow at a rate below $Q=0.4 \mu \mathrm{l} / \mathrm{h}$ of cell culture media through the chamber.

In Fig. 3(a) we show a snapshot of the aligned SaOs-2 cells at the desired nodal positions and as shown in Fig. 3(b) aligned primary neurons, after readjusting the SAW wavelength
$\left(\lambda_{\mathrm{SAW}}=25 \mu \mathrm{m}\right)$ to their diameter $(D \sim 7.7 \pm 0.8 \mu \mathrm{m})$. This serves as starting point to investigate the adhesion and growths of the patterned cells.

After the initial acoustically induced alignment, we allowed the SaOs- 2 cells to adhere on the surface. To do so, the applied power level was decreased to $P_{\mathrm{IN}}=15 \mathrm{dBm}$ or less to still gently hold the cells at the desired positions but at the same time let them interact with the substrate and then ultimately attach to the protein-coated surface. Within about $1 \mathrm{~h}$ after initiating this attachment procedure, they began to start spreading on the substrate, but still being pressure captured at the nodal positions. After the cells adhered, they express their continued well-being by continuing typical cell growth dynamics over at least $1 \mathrm{~h}$ of SSAW, as being illustrated in Fig. 3(c). In contrast to previously published reports [11], we could thus successfully show that SSAW-controlled adhesion on the timescale of several hours is in fact feasible and obviously is compatible with various types of living cells, including the very sensitive neurons.

\section{NEURON ACTIVITY AFTER DIRECTED ADHESION}

We now investigate whether the neuronal cell morphology and activity remain unaffected by SSAW guidance and whether the cells continue to grow after adhesion. Because of the smaller diameter of the neurons as compared to the SaOs-2 cells, we now used a PLL-coated SAW chip and a shorter SAW wavelength $\lambda_{\text {SAW }}=25 \mu \mathrm{m}$ as well as a somewhat smaller channel height of only $h=15 \mu \mathrm{m}$ accordingly. After filling the microchannel with fresh nutrient, we dispensed the cell suspension and activated the symmetric SSAW, thus creating the desired standing wave pressure landscape. First, as described above and to initiate the alignment process, we applied a rf signal with $P_{\mathrm{IN}}=21 \mathrm{dBm}$, which we then reduced it to a gentler and lower power of $P_{\mathrm{IN}}=18 \mathrm{dBm}$ after $t=1 \mathrm{~h}$. To maintain and ensure a constant nutrient supply, the medium was automatically exchanged about every minute by superimposing a slow static flow $(Q=0.4 \mu \mathrm{l} / \mathrm{h})$, as described above. We kept the cells under these conditions for $11 \mathrm{~h}$ and watched them grow while taking a micrograph every $5 \mathrm{~min}$. It was clearly seen that the cells in fact again adhered on the substrate surface and also started to grow extensions. In Fig. 4(a) we depict the neuron culture on the SAW chip after these $11 \mathrm{~h}$. Interestingly and as a very positive result, we again observe neurite outgrowth.

The notable finding of the experiments is the indication that this outgrowth seems to preferentially align along the symmetry axis of the SSAW-induced two-dimensional pressure lattice. In Fig. 4(b) we thus show the extracted angular distribution of neurite extensions' outgrowth direction in the culture shown in Fig. 4(a), as being extracted from an analysis of the time series of the images taken. The yellow arrows indicate the SAW propagation directions. Such SAW-driven "alignment" of elongated entities along the minima of the body force landscape has been studied and observed before in our group for carbon nanotubes [19] as well as biopolymers [20] and also seems to work here. However, for neurons, it opens a wealth of opportunities in terms of deliberate neural network manipulation and formation. 
(a)

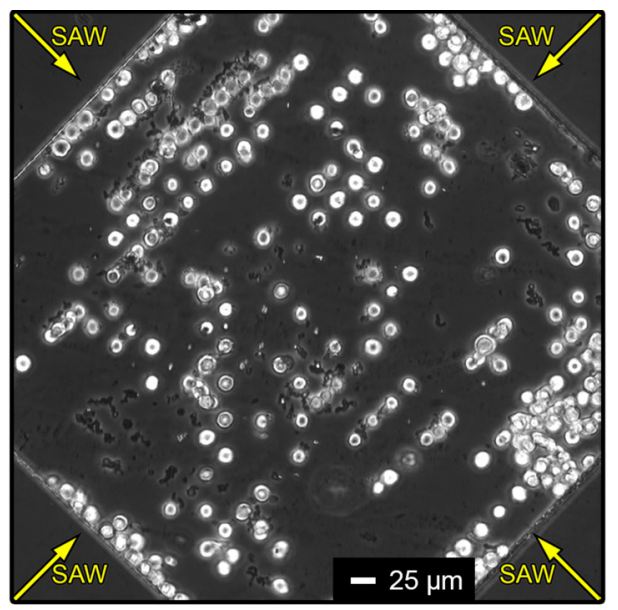

(b)

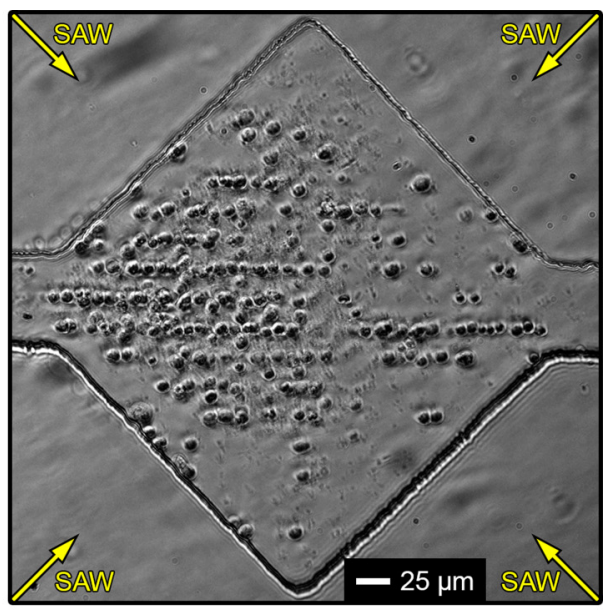

(c)

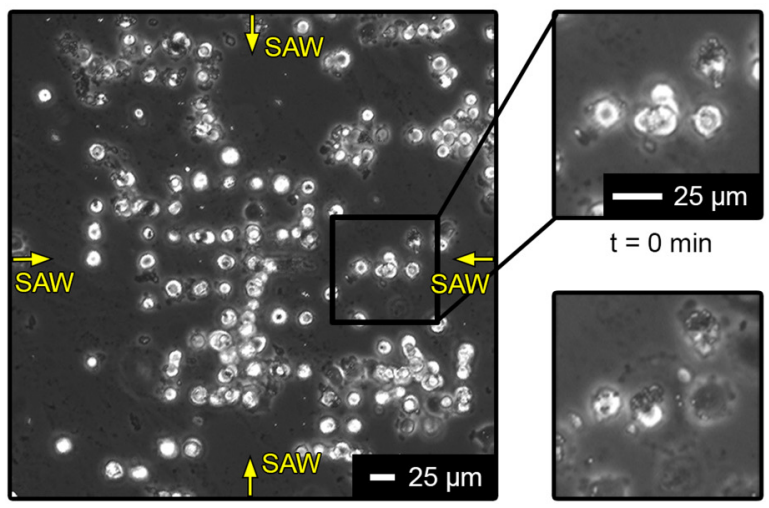

Chamber at $\mathrm{t}=0 \mathrm{~min}$

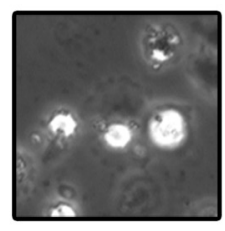

$\mathrm{t}=20 \mathrm{~min}$

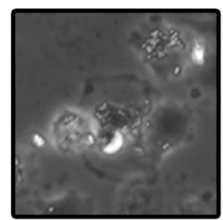

$\mathrm{t}=50 \mathrm{~min}$

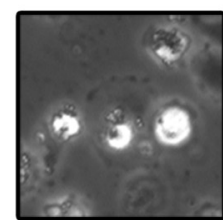

$\mathrm{t}=30 \mathrm{~min}$

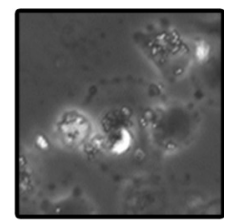

$t=60 \min$

FIG. 3. Adhesion of living cells at nodal positions of the SSAW. (a) SaOs-2 cells in a SSAW field. (b) Primary neurons being laterally aligned by SSAW using a SAW chip with $\lambda_{\mathrm{SAW}}=25 \mu \mathrm{m}$ at a power level of $P_{\mathrm{IN}}=21 \mathrm{dBm}$. (c) SaOs- 2 cell adhesion after successful SSAW-mediated alignment. The insets show a selection of cells moving and spreading as a function of time.

Our preliminary, initial experiments on such guided neuron growth on a widely tunable SAW-activated microfluidic chip will hopefully stimulate further research. In our lab, we are presently performing a series of experiments based on our findings: if we decrease the SAW and thus the pressure amplitude for one of the two IDT pairs, a quasi-one-dimensional standing wave is created, and along the same lines as described in Fig. 4(b) we claim that the neurites will then grow along the nodes in a one-dimensional predetermined confinement. The direction of this confinement and hence the direction and length of the neurite outgrowth is then expected to be rotated by $90^{\circ}$ for our simplest four IDT chip or even in smaller (a)

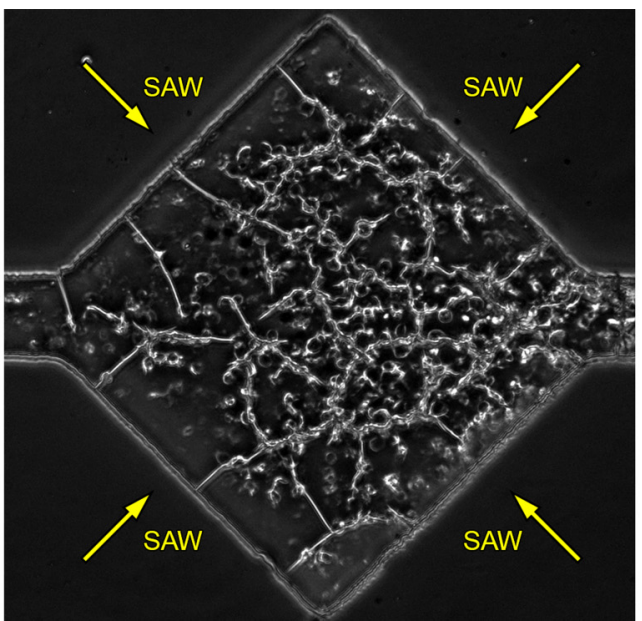

(b)

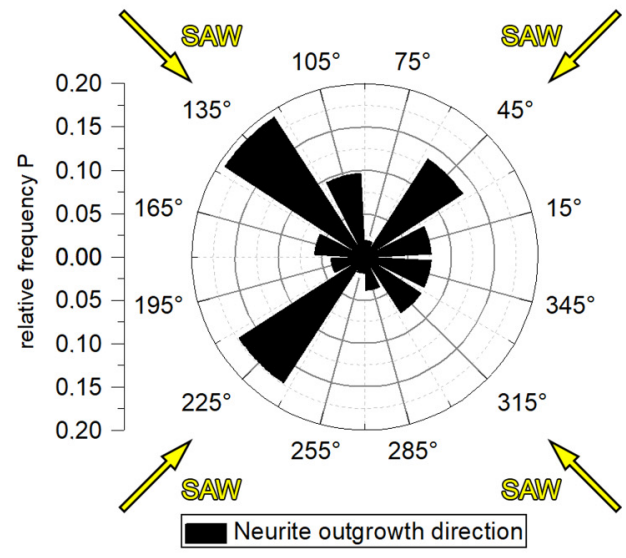

FIG. 4. (a) Neurons after the SSAW at $P_{\mathrm{IN}}=18 \mathrm{dBm}$ was activated for $t=11 \mathrm{~h}$. The adhered neurons even started growing extensions. (b) Relative frequency $P$ of the outgrowth direction. 
angles by a more sophisticated design of transducers. This would allow for a deliberate steering of neurites and a tailored intercell connectivity! These bold but totally feasible ideas of course have to be proven in further experiments including the investigation of the underlying mechanisms in greater detail and will be published elsewhere.

\section{CONCLUSION}

In summary, we have presented a scope of the application of SAW-based microfluidics towards the creation of artificial neural networks. We have shown that we are able to define spatiotemporal, tailored dynamic acoustic force fields in a fluid and a respective acoustic potential landscape being capable of manipulating primary neuron cells. We could confirm that SAWs obviously do not harm the cells but are compatible with successful adherence to the culture surface at the predefined and desired positions. The sensitive neurons do not only survive the SAW treatment, but continue to grow and extend long neurites for at least 4 days. Our experimental findings thus give us the confidence to now employ our SAW technique for the defined growth of neural networks on a chip. We envision creating a pattern of single neurons and plan to employ the finetuning capabilities of SSAW potential landscapes to potentially even direct the subsequent growth of neural extensions. Based on these principles, we hope to be able to control the direction of neural outgrowth without the need of physical or mechanical obstacles or chemical perturbations and thus to overcome remaining persistent limitations for controlled neural networks. In combination with multi-electrode arrays, these cell networks could then be stimulated while simultaneously recording the neural electrical activity. A given network topology could then be taught to perform a task (such as classification) by adapting the synaptic weights in response to coincident firing between the pre- and postsynaptic neurons (spike-dependent timing plasticity). Our approach is in principle compatible with various methods enabling further study and precise stimulation of well-defined neural circuits [21,22]. Time will tell.

\section{ACKNOWLEDGMENTS}

This work has been sponsored by the cluster of excellence "Nanosystems Initiative Munich, NIM," BaCaTeC, the Bavaria California Technology Center, a California NanoSystems Institute Challenge Grant, and Santa Barbara Cottage Hospital Foundation grant. We wish to express our gratitude to the NRI Cell Culture Core Facility, Andrej Kamenac and Lukas Schnitzler for enlightening discussions, Linc Johnson for microscope access, Ken Kosik for primary rat cells, and Melanie Stamp, Cassidy Arnold, and Aaron Bluestone for technical assistance.
[1] P. Fromherz, A. Offenhäusser, T. Vetter, and J. Weis, Science 252, 1290 (1991).

[2] P. Bonifazi and P. Fromherz, Adv. Mater. 14, 1190 (2002).

[3] P. Fromherz, Chem. Phys. Chem. 3, 276 (2002).

[4] P. Fromherz, H. Schaden, and T. Vetter, Neurosci. Lett. 129, 77 (1991).

[5] C. Xie, L. Hanson, W. Xie, Z. Lin, B. Cui, and Y. Cui, Nano Lett. 10, 4020 (2010).

[6] A. Wixforth, in 2009 IEEE International Frequency Control Symposium Joint with the 22nd European Frequency and Time forum, (IEEE, 2009), pp. 778-783.

[7] T. Franke, S. Braunmuller, L. Schmid, A. Wixforth, and D. A. Weitz, Lab Chip 10, 789 (2010).

[8] R. W. Rambach, V. Skowronek, and T. Franke, RSC Adv. 4, 60534 (2014).

[9] Y. Q. Fu, L. Garcia-Gancedo, H. F. Pang, S. Porro, Y. W. Gu, J. K. Luo, X. T. Zu, F. Placido, J. I. B. Wilson, A. J. Flewitt, and W. I. Milne, Biomicrofluidics 6, 024105 (2012).

[10] D. J. Collins, B. Morahan, J. Garcia-Bustos, C. Doerig, M. Plebanski, and A. Neild, Nat. Commun. 6, 8686 (2015).

[11] F. Guo, P. Li, J. B. French, Z. Mao, H. Zhao, S. Li, N. Nama, J. R. Fick, S. J. Benkovic, and T. J. Huang, Proc. Natl. Acad. Sci. USA 112, 43 (2014).

[12] L. Y. Yeo and J. R. Friend, Annu. Rev. Fluid Mech. 46, 379 (2014).
[13] L. Rayleigh and J. W. Strutt, Proc. London Math. Soc. 17, 4 (1885).

[14] X. Ding, S.-C. S. Lin, B. Kiraly, H. Yue, S. Li, I.-K. Chiang, J. Shi, S. J. Benkovic, and T. J. Huang, Proc. Natl. Acad. Sci. USA 109, 11105 (2012).

[15] Z. Wang and J. Zhe, Lab Chip 11, 1280 (2011).

[16] S. M. Naseer, A. Manbachi, M. Samandari, P. Walch, Y. Gao, Y. S. Zhang, F. Davoudi, W. Wang, K. Abrinia, J. M. Cooper, A. Khademhosseini, and S. R. Shin, Biofabrication 9, 15020 (2017).

[17] R. H. Tancrell, M. B. Schulz, H. H. Barrett, L. Davis, and M. G. Holland, Proc. IEEE 57, 1211 (1969).

[18] M. S. Brugger, M. E. M. Stamp, A. Wixforth, and C. Westerhausen, Biomater. Sci. 4, 1092 (2016).

[19] C. J. Strobl, C. Schäflein, U. Beierlein, J. Ebbecke, and A. Wixforth, Appl. Phys. Lett. 85, 1427 (2004).

[20] S. W. Schneider, S. Nuschele, A. Wixforth, C. Gorzelanny, A. Alexander-Katz, R. R. Netz, and M. F. Schneider, Proc. Natl. Acad. Sci. USA 104, 7899 (2007).

[21] P. K. Thanos, L. Robison, E. J. Nestler, R. Kim, M. Michaelides, M. K. Lobo, and N. D. Volkow, J. Neurosci. 33, 6343 (2013).

[22] J. L. Carvalho-de-Souza, B. I. Pinto, D. R. Pepperberg, and F. Bezanilla, Biophys. J. 114, 283 (2018). 